Check for updates

Cite this: Chem. Commun., 2020, 56, 10066

Received 10th June 2020,

Accepted 22nd July 2020

DOI: $10.1039 / \mathrm{d} 0 \mathrm{cc} 04064 a$

rsc.li/chemcomm

\section{Collective enantioselective total synthesis of (+)-sinensilactam A, (+)-lingzhilactone B and $(-)$-lingzhiol: divergent reactivity of styrene $\dagger$}

\author{
Da-Wei Zhang, ${ }^{\text {ab }}$ Hui-Lan Fan, ${ }^{\text {ab }}$ Wenzhao Zhang, ${ }^{c}$ Cheng-Ji Li, ${ }^{\text {ab }}$ \\ Sanzhong Luo (D)*cd and Hong-Bo Qin (D) *a
}

The collective total synthesis of (+)-sinensilactam A, (+)-lingzhilactone $B,(+)$-lingzhilactone $C$ and (-)-lingzhiol has been accomplished from a common epoxide intermediate 9. Chemoselective epoxy opening with either an aryl or alkene moiety of styrene led to different carbon skeletons, which can be advanced to a divergent and concise total synthesis of four meroterpenoids.

During the past 7 years, Ganoderma meroterpenoids have attracted increasing attention among the phytochemical community. With meroterpenoids being increasingly identified, this type of molecule has become the third most abundant constituent of Ganoderma. Sinensilactam A, lingzhilactone B and lingzhiol, isolated from the genus Ganoderma by Cheng, ${ }^{1-3}$ are reno-protective by inhibition of Smad3 phosphorylation in TGF- $\beta 1$-induced cellular assays. In 2018, Yang's group reported the first racemic total synthesis of sinensilactam A using rhodium catalyzed [3+2] cycloaddition as the key step. ${ }^{4}$ However, the synthesis required 18 steps involving harsh conditions such as high pressure $(14 \mathrm{MPa})$ and long-term heating $\left(150{ }^{\circ} \mathrm{C}\right.$, $48 \mathrm{~h}$ ). A concise synthetic route under mild conditions is still highly desirable. On the other hand, we and others have previously reported the total syntheses of lingzhiol and lingzhilactone B. ${ }^{5-12,27}$ In this communication, we have reported a distinctive strategy for the collective synthesis of four meroterpenoids, (+)-sinensilactam A, (+)-lingzhilactone B, (+)-lingzhilactone $\mathrm{C}$ and (-)-lingzhiol. The key step involves non-metal promoted selective

\footnotetext{
${ }^{a}$ State Key Laboratory of Phytochemistry and Plant Resources in West China, Kunming Institute of Botany, Chinese Academy of Sciences, Yunnan Key Laboratory of Natural Medicinal Chemistry, Kunming 650201, P. R. China. E-mail: qinhongbo@mail.kib.ac.cn

${ }^{b}$ University of Chinese Academy of Sciences, Beijing 100049, P. R. China ${ }^{c}$ Institute of Chemistry, Chinese Academy of Sciences, Beijing, 100190, China ${ }^{d}$ Center of Basic Molecular Science, Department of Chemistry, Tsinghua University, Beijing 100084, China.E-mail: luosz@tsinghua.edu.cn

$\dagger$ Electronic supplementary information (ESI) available. CCDC 1888466 and 1888467. For ESI and crystallographic data in CIF or other electronic format see DOI: $10.1039 / \mathrm{d} 0 \mathrm{cc} 04064 \mathrm{a}$
}

nucleophilic opening of epoxide by an alkene moiety of styrene, which has not been explored in total synthesis, though a metalcatalyzed reaction of epoxide with styrene is known. ${ }^{13-15}$

Sinensilactam A can be obtained from dimethyl lingzhilactone B 15 by annulation with 5-hydroxy-2-pyrrolidinone. Lingzhilactone $\mathrm{B}$ has one more carbon at $\mathrm{C} 8$ ' compared to lingzhiol; therefore, styrene is a reasonable equivalent of aryl ketone because it can be either oxidatively cleaved into a dicarbonyl compound, or oxidized into aryl ketone (Fig. 1). The retrosynthetic analysis is outlined below. Ketoaldehyde 15 can be obtained by the ozonolysis of styrene 12, which could be synthesized through an epoxy ring opening reaction with the double-bond of styrene 9. Alternatively, the epoxy ring opening by the aryl moiety would lead to lingzhiol. Styrene 9 could be obtained sequentially by the alkylation of ethyl 2-oxocyclopentane carboxylate with aryl acetyl chloride, twice Wittig reaction, regioselective allylic oxidation and the epoxidation of allylic alcohol.

We started our total synthesis with the assembly of the chiral intermediate 9 (Scheme 1). The alkylation of 2,5-dimethoxyphenylacetyl chloride 1 with ethyl 2-oxocyclopentanecarboxylate proceeded smoothly to give diketone 2 in $94 \%$ yield. A resolution by baker's yeast only recovered the starting material. Therefore, we adopted a detour of a kinetic resolution of the known alkyne

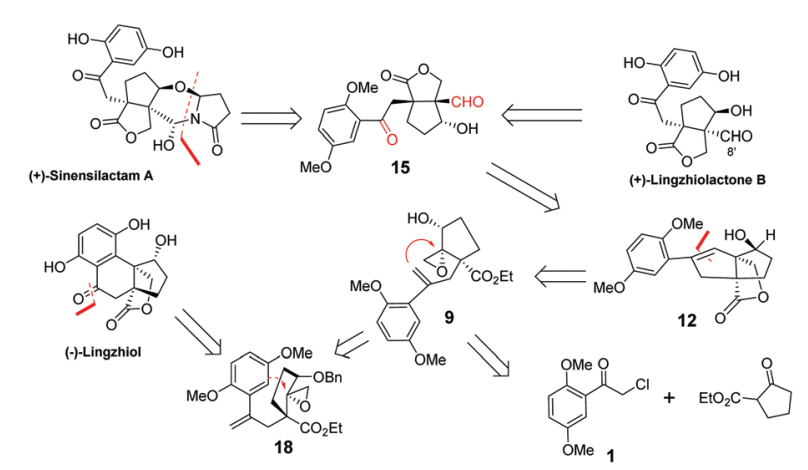

Fig. 1 Structures of several meroterpenoids and retrosynthetic analysis. 

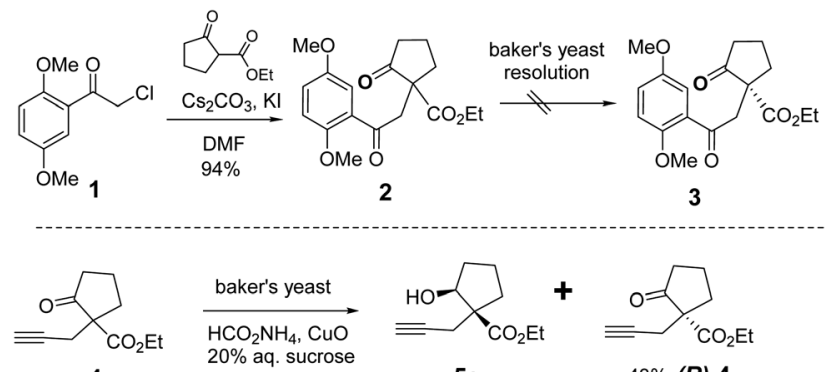

4

$5 \mathbf{a}$

$43 \%(R)-4$
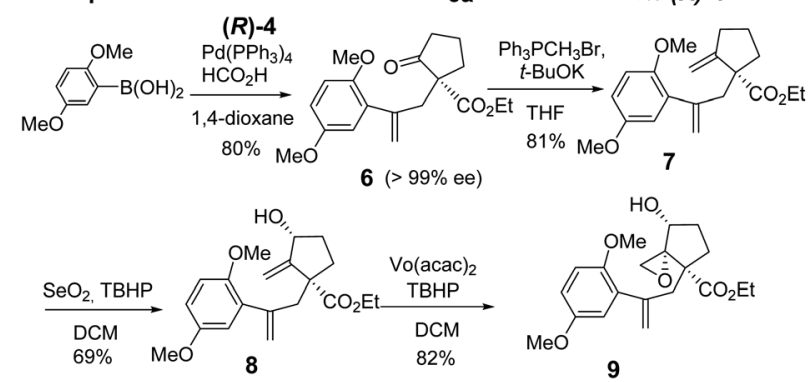

Scheme 1 Construction of a chiral common intermediate 9

ketoester 4. The absolute configuration of the recovered ketone was determined to be $R$ by its transformation into the known (-)-lingzhiol. ${ }^{1}$ The addition of 2,5-dimethoxyphenylboronic acid to chiral alkyne $(R)-4$ gave styrene 6 in $80 \%$ yield. ${ }^{16}$ The Wittig reaction of ketone 6 with the ylide generated from $t$-BuOK and triphenyl methyl bromide in THF afforded diene 7 in $81 \%$ yield as an optically pure enantiomer. ${ }^{17}$ Regioselective allylic oxidation with selenium dioxide and $t$-butyl hydroperoxide converted 7 into allylic alcohol 8 in $69 \%$ yield. The regioselectivity can be attributed to the steric hindrance of quaternary carbon and the cis-stereoselectivity possibly arose from the chelation of selenium with the ester group. Next, Sharpless epoxidation ${ }^{18}$ produced cis-epoxy secondary alcohol 9 regioselectively in $82 \%$ yield, which is an advanced intermediate with versatile reactivity.

We embarked on the first asymmetric synthesis of sinensilactam A and lingzhilactone B because their enantiomers have different bioactivities. ${ }^{3}$ To accomplish this, the double bond instead of the aryl ring should preferentially engage in nucleophilic attack (Scheme 2). A similar aryl-epoxide opening has previously been reported, and it was found that the aryl attack occurs from the opposite face of epoxide. ${ }^{6,10}$ In our case, the space-demanding cis-geometry of the neighboring hydroxyl group may reinforce this aryl preference. To overturn this preference, we sought a stereoelectronic approach by oxidizing the cyclopentanol to cyclopentanone. By doing so, the reactivity of the epoxide moiety is enhanced due to the electronwithdrawing effect, facilitating the less-nucleophilic alkeneattack. In addition, the flattened cyclopentanone ring would disfavor the aryl attack as a result of steric repulsion between the approaching aryl methoxy group and the ketone moiety.

To our delight, after oxidation into ketone with DMP, the desired styrene-addition adduct $\mathbf{1 1}$ was obtained when treated with $\mathrm{BF}_{3} \cdot \mathrm{Et}_{2} \mathrm{O}$. Lactone 12 was obtained through the reduction of ketone with $\mathrm{NaBH}_{4}$, followed by lactonization upon treatment

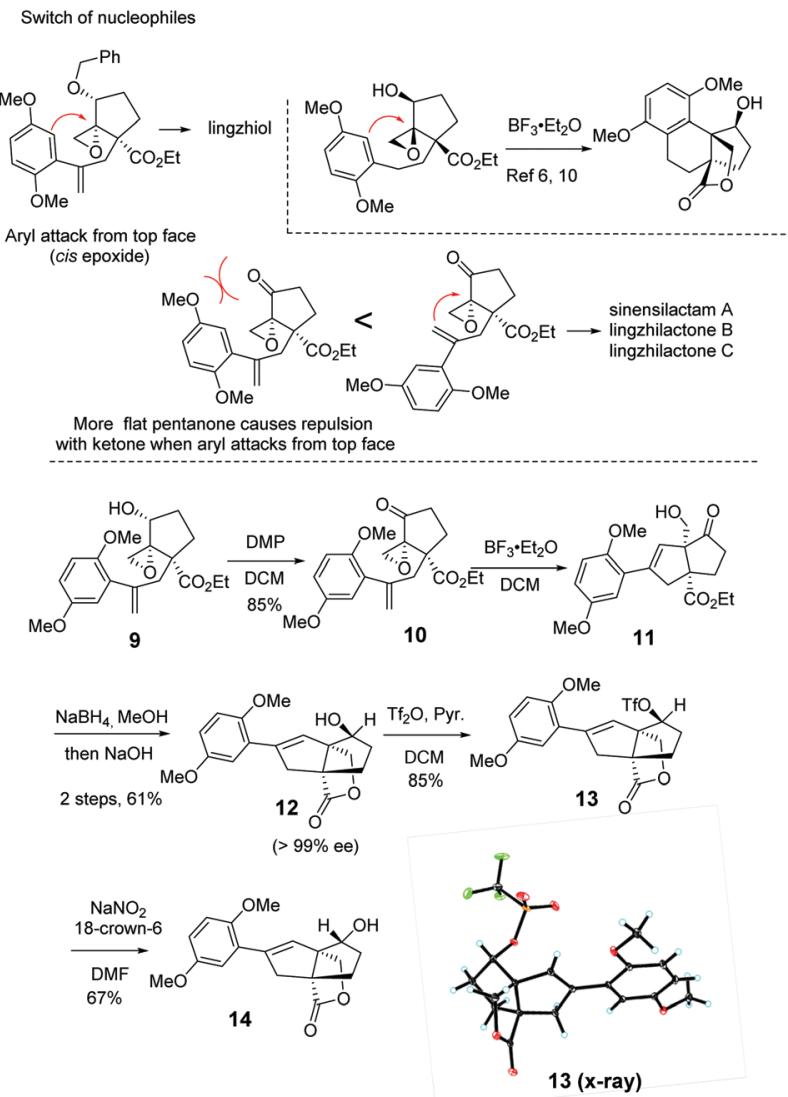

Scheme 2 Construction of bicyclic styrene 12

with $\mathrm{NaOH}$ in $\mathrm{MeOH}$. The hydroxyl group was trans to the lactone due to the directing effect of the adjacent hydroxyl group (Scheme 2, 11) during reduction. Considering the relative configurations of sinensilactam A and lingzhilactone B, the inversion of the configuration of the hydroxyl group is needed. Our extensive examination of the commonly used Mitsunobu conditions was futile, ${ }^{19-23}$ and no inversion occurred. Eventually, when compound 12 was treated with $\operatorname{Tf}_{2} \mathrm{O}$ and pyridine, triflate 13 was converted into cis isomer 14 in the presence of $\mathrm{NaNO}_{2}$ and 18crown- $6 .{ }^{24}$ The structure of $\mathbf{1 3}$ was unambiguously determined by single-crystal X-ray diffraction analysis.

From styrene lactone 14, the total synthesis was achieved following Yang's procedure (Scheme 3). Dihydroxylation and the subsequent $\mathrm{Pb}(\mathrm{OAc})_{4}$-mediated cleavage of the resulting diol proceeded smoothly to afford the key intermediate 15, which was then deprotected with $\mathrm{BBr}_{3}$ to complete the total synthesis of $(+)$-lingzhilactone B. The further protection of aldehyde into acetal gave (+)-lingzhilactone C. From ketoaldehyde 15, we tried to utilize 5-ethoxy-2-pyrrolidinone, instead of 5-hydroxy-2-pyrrolidinone, in order to improve the yield. As is well-known, the in situ generation of an $\mathrm{N}$-acyliminium intermediate from 5-ethoxy-2-pyrrolidinone proceeded much faster at lower temperature $\left(1 \mathrm{~h} v s .5 \mathrm{~h}, 0{ }^{\circ} \mathrm{C} v s .40{ }^{\circ} \mathrm{C}\right)$. Unfortunately, we encountered serious issues during purification as its $R_{\mathrm{f}}$ value was the same as that of the product. Finally, the total synthesis of (+)-sinensilactam A was achieved by the condensation of $\mathbf{1 5}$ with 


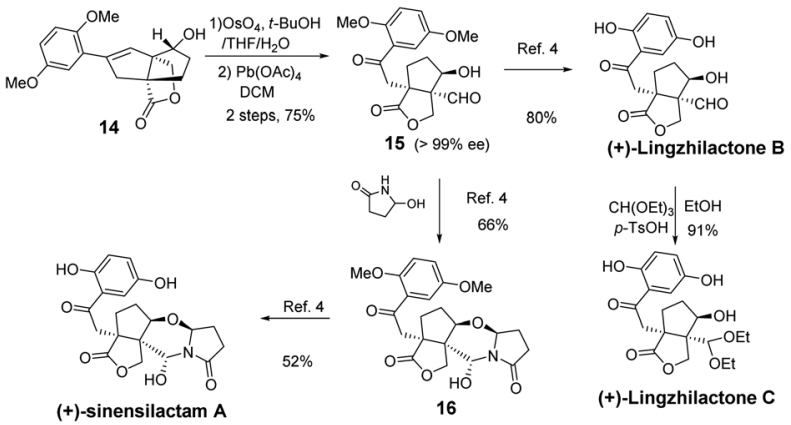

Scheme 3 Completion of the synthesis of $(+)$-sinensilactam A.

5-hydroxy-2-pyrrolidinone (66\% yield) and subsequent demethylation with $\mathrm{BBr}_{3}(52 \%$ yield). The synthetic samples have rotations of $+50.3(c 0.07, \mathrm{MeOH})[\text { literature value: }+35.7(c 0.11, \mathrm{MeOH})]^{3}$ for $(+)$-sinensilactam A, +154.8 ( $c 0.08$, acetone) for $(+)$-lingzhilactone $\mathrm{B}$ and +114.3 (c 0.06, DMSO) for $(+)$-lingzhilactone $\mathrm{C}$.

Next, the total synthesis of lingzhiol was investigated from epoxy styrene 9 (Scheme 4). The hydroxyl group of 9 was protected as benzyl ether 17 in $75 \%$ yield, with the purpose of preventing the undesired semipinacol rearrangement under Lewis acid catalysis. ${ }^{25}$ Benzyl ether 17 underwent an arylepoxide opening reaction to give the expected tetracyclic styrene 18 by TMSOTf catalysis. ${ }^{6}$ An initial experiment with $\mathrm{BF}_{3}$. $\mathrm{Et}_{2} \mathrm{O}$ gave the desired product in $40 \%$ isolated yield. After further screening the Lewis acid and temperature, the optimal $51 \%$ isolated yield was obtained with 2.5 equiv. TMSOTf in $\mathrm{DCM}$ at $0{ }^{\circ} \mathrm{C}$. Under the same conditions, $\mathrm{BF}_{3} \cdot \mathrm{Et}_{2} \mathrm{O}$ afforded the desired product in $45 \%$ yield. No alkene-attack product was observed in these cases. Routine $\mathrm{OsO}_{4}$-catalyzed dihydroxylation and subsequent oxidative cleavage of diol with $\mathrm{NaIO}_{4}$ afforded ketoester 19 in $74 \%$ yield. In this way, we took a detour of benzylic oxidation, which was usually not effective. ${ }^{8}$
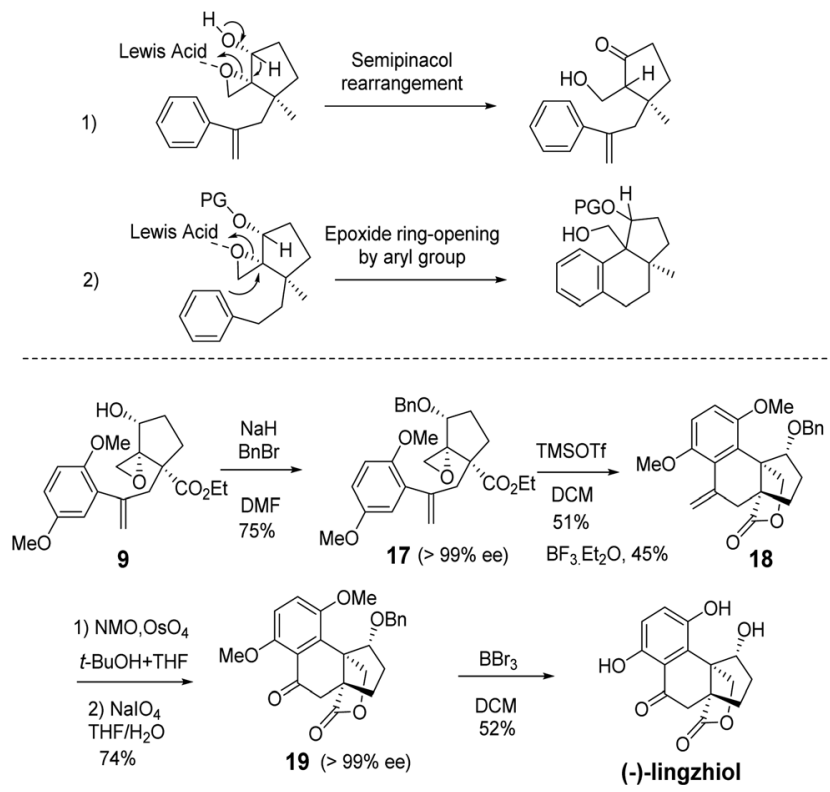

Scheme 4 Total synthesis of (-)-lingzhiol.
Finally, the global deprotection of dimethoxy and benzyloxy groups furnished (-)-lingzhiol in $52 \%$ yield. Our synthesis took 10 steps and the overall yield was $2.3 \%$.

The synthetic strategy is straightforward and the conditions are mild. The synthetic sample has a rotation of -60.7 ( $c 0.12$, $\mathrm{MeOH})$ [literature value: $-94.2(c 0.36, \mathrm{MeOH})] .{ }^{1}$ Our NMR data of the synthetic samples are all in agreement with those of the literature.

To demonstrate the utility of our synthetic strategy, the $(+)$-sinensilactam A isomer and (+)-epi-lingzhilactone $\mathrm{B}$, which are C6 $^{\prime}$ epimers of natural products, were also prepared following similar operations (Scheme 5). From styrene 12, a similar transformation of double bonds afforded 20 in $78 \%$ yield. It was then demethylated to give (+)-epi-lingzhilactone $\mathrm{B}$ in good yield $(81 \%)$. When similar operations of pyrrolidinone installation and demethylation were performed, the $(+)$-sinensilactam A isomer was isolated in a comparable yield. The structure of 21 was confirmed by single-crystal X-ray diffraction analysis.

Finally, we sought an alternative asymmetric synthesis of the key chiral intermediate 3 using the state-of-the-art enantioselective photocatalytic process. Based on our previously developed chiral primary amine catalyzed photolytic alkylation reaction, ${ }^{23}$ the catalytic asymmetric synthesis of the chiral quaternary diketoester 3 , which can be readily transformed into 7, was investigated (Scheme 6). Under the optimal conditions, ${ }^{26}$ up to $99 \%$ ee was realized with $71 \%$ isolated yield at $100 \mathrm{mg}$ scale. It can be transformed into key diene intermediate 7 under similar Wittig reaction conditions, possibly shortening the whole synthesis by two steps.

In summary, we have developed a collective strategy for the concise synthesis of four meroterpenoids based on the divergent reactivity of styrene, which is significantly different from the widely used semipinacol rearrangement strategy in the syntheses of similar meroterpenoids. A regioselective epoxy ring-opening reaction with the double bond of styrene provided the key lactone styrene 12, leading to the total synthesis of (+)-sinensilactam A for the first time in 14 steps and $1.1 \%$ overall yield. Starting with the same intermediate, (+)-lingzhilactone B was obtained in 13 steps and $2.7 \%$ overall yield. On the other hand, the key reaction to (-)-lingzhiol features a one-step construction of a 5/5/6/6 ring system by simultaneous epoxy ring-opening and lactonization promoted
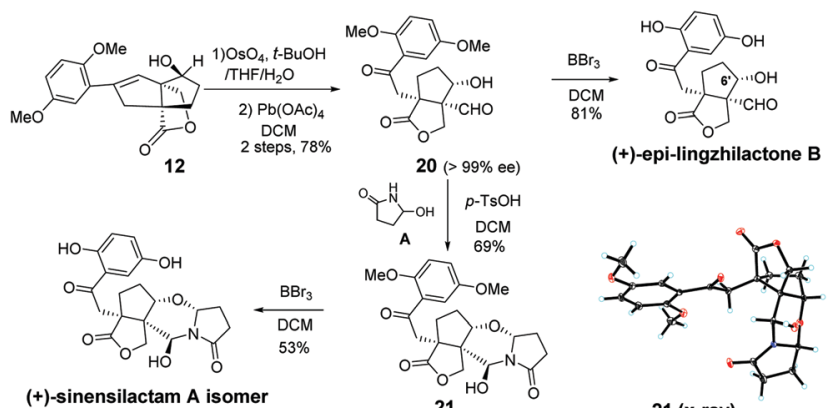

(+)-sinensilactam A isome

21 (x-ray)

Scheme 5 Synthesis of the two isomers. 


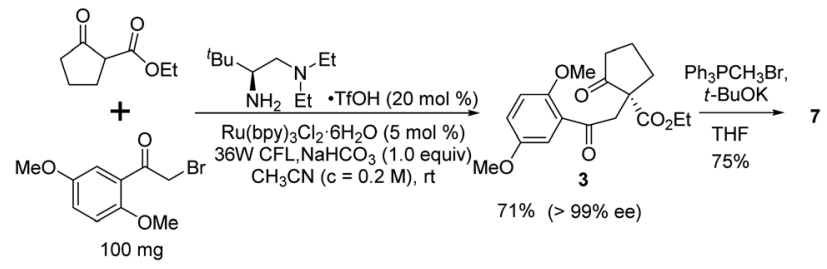

Scheme 6 Catalytic asymmetric construction of the quaternary stereocenter.

by TMSOTf in 10 steps with an overall yield of $2.3 \%$. The current strategy provides a detour from the problematic benzylic oxidation in the total synthesis of this series of natural products. Finally, a catalytic asymmetric synthesis of the key chiral intermediate 3 was also achieved by using the established chiral primary aminocatalytic protocol. It should be noted that the construction of all-carbon quaternary centers is quite efficient under rather mild conditions, and may have further applications in total synthesis.

This work was financially supported by the High End Talent Program of Yunnan Province to Dr H.-B. Q. (2015HA028), the NSFC (21372229, 21861132003 and 21672217), the State Key Laboratory of Phytochemistry and Plant Resources in West China (Y83802A), and the Kunming Institute of Botany, CAS.

\section{Conflicts of interest}

There are no conflicts to declare.

\section{Notes and references}

1 Y.-M. Yan, J. Ai, L. L. Zhou, A. C. K. Chung, R. Li, J. Nie, P. Fang, X.-L. Wang, J. Luo, Q. Hu, F.-F. Hou and Y.-X. Cheng, Org. Lett., 2013, 15, 5488-5491.

2 Y.-M. Yan, X.-L. Wang, L.-L. Zhou, F.-J. Zhou, R. Li, Y. Tian, Z.-L. Zuo, P. Fang, A. C. K. Chung and F.-F. Hou, J. Ethnopharmacol., 2015, 176, 385-393.
3 Q. Luo, L. Tian, L. Di, Y.-M. Yan, X.-Y. Wei, X.-F. Wang and Y.-X. Cheng, Org. Lett., 2015, 17, 1565-1568.

4 W. Shao, J. Huang, K. Guo, J. Gong and Z. Yang, Org. Lett., 2018, 20, 1857-1860.

5 R. Long, J. Huang, W. Shao, S. Liu, Y. Lan, J. Gong and Z. Yang, Nat. Commun., 2014, 5, 5707.

6 D. Chen, H.-M. Liu, M.-M. Li, Y.-M. Yan, W.-D. Xu, X.-N. Li, Y.-X. Cheng and H.-B. Qin, Chem. Commun., 2015, 51, 14594-14596.

7 D. Chen, W. D. Xu, H. M. Liu, M. M. Li, Y. M. Yan, X. N. Li, Y. Li, Y. X. Cheng and H. B. Qin, Chem. Commun., 2016, 52, 8561-8564.

8 K. Sharmah Gautam and V. B. Birman, Org. Lett., 2016, 18, 1499-1501.

9 L.-M. Mehl and M. E. Maier, J. Org. Chem., 2017, 82, 9844-9850.

10 R. Rengarasu and M. E. Maier, Asian J. Org. Chem., 2017, 6, 108-117.

11 X. Li, X. Liu, X. Jiao, H. Yang, Y. Yao and P. Xie, Org. Lett., 2016, 18, 1944-1946.

12 D. Chen, X.-M. Li, H.-M. Liu, M.-M. Li, Y.-X. Cheng and H.-B. Qin, Tetrahedron Lett., 2016, 57, 2877-2879.

13 Y. Ikeda, H. Yorimitsu, H. Shinokubo and K. Oshima, Adv. Synth. Catal., 2004, 346, 1631-1634.

14 G. P. Cerai and B. Morandi, Chem. Commun., 2016, 52, 9769-9772.

15 S. H. Teng, M. E. Tessensohn, R. D. Webster and J. S. Zhou, ACS Catal., 2018, 8, 7439-7444.

16 C. H. Oh, H. H. Jung, K. S. Kim and N. Kim, Angew. Chem., Int. Ed., 2003, 42, 805-808.

17 R. Rengarasu and M. E. Maier, Asian J. Org. Chem., 2017, 6, 108-117.

18 K. B. Sharpless and R. C. Michaelson, J. Am. Chem. Soc., 1973, 95, 6136-6137.

19 P. O'Brien, C. M. Rosser and D. Caine, Tetrahedron Lett., 2003, 44, 6613-6615.

20 F. Mazraati Tajabadi, R. H. Pouwer, M. Liu, Y. Dashti, M. R. Campitelli, M. Murtaza, G. D. Mellick, S. A. Wood, I. D. Jenkins and R. J. Quinn, J. Med. Chem., 2018, 61, 6609-6628.

21 J. A. Davy, J. W. Mason, B. Moreau and J. E. Wulff, J. Org. Chem., $2012,77,6332-6339$.

22 S.-Z. Jiang, T. Lei, K. Wei and Y.-R. Yang, Org. Lett., 2014, 16, 5612-5615.

23 P. Yates, R. S. Grewal, P. C. Hayes and J. F. Sawyer, Can. J. Chem., 1988, 66, 2805-2815.

24 A. Nakazato, K. Sakagami, A. Yasuhara, H. Ohta, R. Yoshikawa, M. Itoh, M. Nakamura and S. Chaki, J. Med. Chem., 2004, 47, $4570-4587$.

25 Z. L. Song, C. A. Fan and Y. Q. Tu, Chem. Rev., 2011, 111, 7523-7556.

26 Y. Zhu, L. Zhang and S. Luo, J. Am. Chem. Soc., 2014, 136, 14642-14645.

27 P. S. Riehl, A. D. Richardson, T. Sakamoto and C. S. Schindler, Org. Lett., 2020, 22, 290-294. 